\title{
Bcr-Abl fusion sequences do not induce immune responses in mice when administered in mouse polyomavirus based virus-like particles
}

\author{
VERONIKA HRUSKOVÁ ${ }^{1}$, ALENA MORÁVKOVÁ ${ }^{1}$, KATARINA BABIAROVÁ $^{2}$, \\ VIERA LUDVÍKOVÁ ${ }^{2}$, JAN FRIC ${ }^{1}$, VLADIMÍR VONKA ${ }^{2}$ and JITKA FORSTOVÁ ${ }^{1}$ \\ ${ }^{1}$ Faculty of Science, Charles University in Prague, Vinicná 5, 12844 Prague 2; ${ }^{2}$ Department of Experimental Virology, \\ Institute of Hematology and Blood Transfusion, U Nemocnice 1, 12820 Prague 2, Czech Republic
}

Received June 15, 2009; Accepted August 18, 2009

DOI: 10.3892/ijo_00000441

\begin{abstract}
Mouse polyomavirus-like particles (MPyV-VLPs) carrying inside a fragment of the Bcr-Abl hybrid protein containing the epitope of chronic myeloid leukemia fusion region were prepared. A sequence encoding 171 amino acids covering Bcr-Abl breakpoint was fused to the $\mathrm{C}$-terminal part of VP3 minor protein connecting it to the VP1 capsomeres. Chimeric particles, the Bcr-Abl VLPs, were tested for their ability to induce Bcr-Abl specific immune response in mice after their intranasal (i.n.) or intraperitoneal (i.p.) administration without any other adjuvants. Bcr-Abl VLPs induced strong anti-VP1 immune response in both i.n. and i.p. immunized mice. As expected, neither IgG nor IgM anti-Bcr-Abl specific antibodies were detected in the sera of immunized animals. Surprisingly, no specific CTL (cytotoxic T-lymphocyte) activity was proved using two different methods (in vitro cytotoxicity assay with CFSE-labeled target cells and highly sensitive cytotoxicity assay using MHC class I Bcr-Abl specific pentamers). In addition, no proliferative response of T-cells of i.n. immunized mice after in vitro restimulation with antigen-pulsed bone marrow-derived dendritic cells was observed. Taken together, Bcr-Abl breakpoint epitopes appeared to be weak immunogens and even MPyV-VLPs did not provide sufficient adjuvant ability to support induction of immune responses specific to Bcr-Abl fusion zone epitope.
\end{abstract}

\section{Introduction}

Chronic myeloid leukemia (CML) is characterized by a reciprocal translocation between chromosomes 9 and 22 .

Correspondence to: Dr Jitka Forstová, Faculty of Science, Charles University in Prague, Vinicná 5, 12844 Prague 2, Czech Republic E-mail: jitkaf@natur.cuni.cz

Key words: mouse polyomavirus VLPs, Bcr-Abl epitope, chronic myeloid leukemia
As a consequence of translocation, parts of the abl gene become fused with breakpoints of the bcr gene. Resulting gene products, Bcr-Abl fusion proteins, contain a new sequence of amino acids in the breakpoint region which is not present in any other known proteins. The most frequent mRNAs contain junctions $\mathrm{b} 2 \mathrm{a} 2$ or $\mathrm{b} 3 \mathrm{a} 2$ (in the second or third bcr exones and in the second abl exon) resulting in the expression of fusion protein $\mathrm{p} 210^{\mathrm{Bcr}-\mathrm{Abl}}$. The newly produced fusion protein is characterized by abnormally high activity of tyrosine kinase mediated by the SH1 domain of the Abl protein (1). As the junctional sequences of Bcr-Abl are only expressed in leukemic cells, they might be promising tumor specific targets for the immunotherapy. Both human studies in vitro or in vivo and in vivo mouse model studies have demonstrated that leukemia associated antigens such as the peptides derived from breakpoint region of fused Bcr-Abl protein may serve for immunotherapy. Rojas et al (2) reported that the administration of peptide vaccines derived from b3a2 gene breakpoint generated $\mathrm{T}$-cell responses in the majority of patients. In a mouse model, chaperon-rich liver cell lysate embedded with MHC restricted $\mathrm{Bcr}-\mathrm{Abl}$ peptide generated potent immunity against pre-established murine leukemia, in contrast to administration of the Bcr-Abl peptide alone or chaperon-rich liver cell lysate without the peptide (3).

VLPs derived from viruses of different viral families are being intensively studied as inducers of immune responses not only against viral capsid proteins themselves but also as stimulators of immune responses against foreign epitopes carried by chimeric VLPs (4-6). Previously, we developed VLPs derived from mouse polyomavirus (7-9). Polyomaviruses are small non-enveloped viruses with $5.3 \mathrm{kbp}$ circular genome DNA. The icosahedral capsid is formed by 72 pentamers of the major structural protein VP1. The minor capsid proteins, VP2 and VP3, are not exposed on the surface of polyomavirus capsids. Their common C-termini interact with the central cavity of VP1 pentamers, while their N-termini are oriented towards the nucleocore. Neither VP2 nor VP3 is required for VLP formation. VP1 produced in insect or yeast cells is able to self-assemble into VLPs in the cell nuclei. VLPs can be formed in vitro from VP1 pentameres produced in $E$. coli $(7,10,11)$. MPyV-VLPs, like native virions, enter 
cells by receptor-mediated endocytosis via VP1 interaction with the ganglioside receptors GD1a or GT1b (12).

MPyV-VLPs are highly immunogenic, enter different types of cells including mouse and human dendritic cells and are able to reach a broad range of mouse tissues after different types of administration $(8,13,14)$. Several studies followed the immune responses evoked by MPyV-VLPs. It was shown that MPyV-VLPs induced both humoral and cellular immune responses and their application protected mice against polyomavirus infection $(15,16)$. Furthermore, exploitation of MPyV-VLPs as carriers of foreign epitopes has been examined. Several types of chimeric VLPs were prepared and used in different immunization protocols (17-19). In some studies, the short epitopes were exposed on the surface of the particle by insertion into the surface loops of the major structural protein VP1.

Previously (13), we studied the model of chimeric MPyVVLPs containing enhanced green fluorescence protein (EGFP) fused with the C-terminal part of the VP3 minor capsid protein inside the particles (EGFP-VLPs). We showed that the particles entered mouse and human dendritic cells efficiently and were processed by both lysosomes and proteasomes. They induced secretion of interleukin 12 (IL-12) by mouse and human dendritic cells in vitro. In the following study (20), we used EGFP-VLPs for immunization of mice. Splenocytes of immunized mice (restimulated in vitro with EGFP pulsed bone marrow derived dendritic cells) exhibited remarkable T-cell proliferative response specific for EGFP and secreted IL-2 and interferon- $\gamma$ (IFN $\gamma$ ). We observed that splenocytes from mice immunized by EGFP-VLPs exhibited stronger T-cell proliferation response than splenocytes from mice immunized by EGFP administered with Freund's adjuvant.

In this study, we were interested whether virus-like particles derived from mouse polyomavirus carrying Bcr-Abl fusion sequences would be able to promote induction of Bcr-Abl specific immune response in mice after their intranasal or intraperitoneal administration.

\section{Materials and methods}

Plasmid construction and generation of recombinant baculoviruses. Sequences of human fusion protein $\mathrm{p} 210^{\mathrm{bcr}-\mathrm{abl}}$ were obtained from pGD210 plasmid (21). Recombinant baculovirus was prepared for production of chimeric MPyV-based VLPs, carrying epitope covering b3a2 breakpoint of Bcr-Abl fusion protein. For preparation of Bcr-Abl VLPs, the plasmid (pFastBacDual-VP1/tVP3), carrying the MPyV genes for the major structural protein, VP1, and the truncated minor structural protein VP3 (tVP3) under the polyhedrin promoter and p10 promoter respectively was kindly provided by $\mathrm{M}$. Marek (unpublished data). Sequence of p210 acids long (aa810-aa981), covering newly created lysine 928 of $\mathrm{b} 3 \mathrm{a} 2$ breakpoint was amplified by PCR. PCR primers were designed to introduce sequences of FLAG epitope upstream of Bcr-Abl sequences for easy detection of inserted amino acids. The PCR fragment was inserted into pFastBacDualVP1/tVP3 to generate the plasmid pFastBacDual-VP1/ $\mathrm{Bcr}-\mathrm{Abl}_{171}$-tVP3, where FLAG-Bcr-Abl sequences were connected in frame with 5-end of tVP3 sequences. The donor plasmid pFastBacDual-VP1/Bcr-Abl ${ }_{171}$-tVP3 was then used for preparation of recombinant baculovirus, according to Invitrogen instructions (22) and O'Reilly et al (23).

Cell cultures. Spodoptera frugiperda (Sf9) cells were grown as monolayer cultures at $27^{\circ} \mathrm{C}$ in TNM-FH medium (Sigma) containing $10 \%$ fetal calf serum (FCS, Gibco) as described by Hink (24). Swiss albino mouse fibroblasts 3 T6 were grown at $37^{\circ} \mathrm{C}, 5 \% \mathrm{CO}_{2}$ in Dulbecco's modified Eagle's medium (DMEM; Sigma) supplemented with $4 \mathrm{mM}$ L-glutamine (Gibco) and 10\% FCS.

Antibodies. The following antibodies were used for immunostaining and as a positive control for detection of antibodies by ELISA: anti-MPyV VP1 mouse monoclonal antibody (7), rabbit polyclonal antibody against the epitope created by the fusion of Bcr and Abl proteins (25), mouse monoclonal anti-Flag antibody (Sigma), mouse monoclonal anti-MPyV VP2/3 antibody (7). Secondary antibodies: Alexa Fluor-488 (green) goat anti-mouse antibody (Molecular Probes) for immunofluorescence staining, goat anti-rabbit IgG antibody conjugated with $10 \mathrm{~nm}$ gold particles for immunoelectron microscopy (British Biocell Int.), goat anti-mouse IgG horseradish peroxidase-conjugated antibody (Bio-Rad) or goat anti-mouse IgM horseradish peroxidase-conjugated antibody (Sigma) for detection of specific antibodies in the sera of mice by ELISA and for Western blot analysis. Rat anti-mouse CD19 antibody conjugated with PE-Cy5 (ProImmune, Oxford, UK), rat anti-mouse CD8 antibody conjugated with FITC (ProImmune, Oxford, UK), mouse anti-mouse MHCII antibody conjugated with FITC (Serotec, Kidlington, UK) and rat anti-mouse CD86 antibody conjugated with R-PE (Molecular Probes) were used for flow cytometric analysis.

Synthetic peptides and MHC class I pentamers. The peptide covering Bcr-Abl fusion region GFKQSSKAL (9-mer BcrAbl peptide), the control HYLSTQSAL peptide (referred as irrelevant peptide), both used for pulsing of dendritic cells, and longer Bcr-Abl peptide (18-mer Bcr-Abl peptide) HSAT GFKQSSKALQRPVA used for control mice immunization were synthesized by Clonestar Peptide Services (Prague, Czech Republic). R-PE labeled murine H2-Kd/GFKQSSKAL MHC class I pentamers were synthesized by ProImmune (Oxford, $\mathrm{UK})$.

Preparation of virus-like particles. Insect cells Sf9 were infected (10 PFU per cell) with recombinant baculovirus carrying the genes for production of VP1 and Bcr-Abl $1_{171}-\mathrm{tVP} 3$ or with baculovirus for production of VP1 only. Cells were harvested $72 \mathrm{~h}$ post infection, lysed and virus-like particles were purified by $\mathrm{CsCl}$ and sucrose gradients as described previously (7). The quality of preparation was examined by SDS-PAGE, Western blotting and by electron microscopy. The amounts of VP1 were determined by Bradford method.

SDS-PAGE and Western blot analysis. Purified chimeric VLPs boiled for $5 \mathrm{~min}$ in Laemmli loading buffer were separated on $10 \%$ SDS-polyacrylamide gels. Proteins were stained with Coomassie brilliant blue to estimate an average number of 


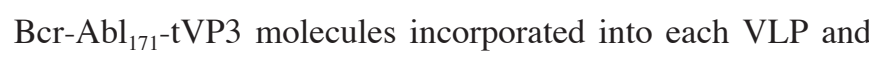
densities of VP1 and Bcr-Abl ${ }_{171}-\mathrm{tVP} 3$ bands were measured. For Western blot analysis, proteins were electro-transferred onto a nitrocellulose membrane (Sigma). The membranes were incubated in 5\% fat-free milk in PBS for $1 \mathrm{~h}$. Subsequent immunostaining with primary and secondary antibodies was carried out by incubation for $1 \mathrm{~h}$ and $30 \mathrm{~min}$ respectively, with intensive washing with PBS after both incubations. Proteins were exposed to X-ray film using chemiluminescence detection system (ECL, Amersham).

Electron and immunoelectron microscopy. For morphology analysis, VLPs were adsorbed on carbon-coated parlodion membrane of electron microscopy (EM) grids and activated with glow discharge. Grids were then washed in redistilled $\mathrm{H}_{2} \mathrm{O}$ and contrasted on a drop of phosphowolframic acid. For immunoelectron microscopy analysis, grids with adsorbed VLPs were incubated with a drop of primary antibody, washed in PBS and exposed to colloidal gold-conjugated secondary antibody. Grids were washed in redistilled $\mathrm{H}_{2} \mathrm{O}$ and finally contrasted by phosphowolframic acid. For detection of Bcr-Abl epitopes, the VLPs were disassembled into pentamers by treatment with $10 \mathrm{mM}$ DTT (Roche) prior to adsorption to uncover epitopes for anti-Bcr-Abl antibody.

Immunofluorescence staining. Cells growing in a 24-well dish on cover slips were transferred on ice and pseudoinfected with VLPs (10 ${ }^{4}$ VLPs per cell). After 30-min adsorption of VLPs on the cell surface $\left(\right.$ at $0{ }^{\circ} \mathrm{C}$ ), cells were either fixed immediately or supplied with $0.5 \mathrm{ml}$ of warmed $\left(37^{\circ} \mathrm{C}\right)$ DMEM with $10 \% \mathrm{FCS}$, incubated for $1 \mathrm{~h}$ at $37^{\circ} \mathrm{C}$ and then fixed. Fixing (after rinsing cells with PBS) was performed with $3 \%$ paraformaldehyde in PBS for $15 \mathrm{~min}$. Cells were further permeabilized using $0.5 \%$ Triton X-100 (Sigma) in PBS for 5 min. Fixed cells were saturated by $0.25 \%$ bovine serum albumin (BSA; Sigma) and $0.25 \%$ gelatin (Sigma) in PBS. Immunostaining with primary and secondary antibodies was carried out by incubation for $1 \mathrm{~h}$ and 30 min respectively, followed by washing with PBS after each incubations.

Mice. Female mice (BALB/c) at the age of 7-10 weeks were used in the experiments. The experiments were approved by the local Animal Ethics Committee.

Immunizations. Mice were divided into groups and immunized intranasally with two doses or intraperitoneally with four doses of chimeric (or wild-type, composed of VP1 only) VLPs in two week intervals. Some groups of animals were immunized with Bcr-Abl peptide (18-mer) twice, first in CFA (complete Freund's adjuvant) second in incomplete Freund's adjuvant. The amount of protein used for immunization was $50 \mu \mathrm{g} /$ dose/mouse. Twelve to 14 days after the last immunization, animals were bled by tail snipping under total anesthesia with Narcotan and then sacrified. Spleens were removed and analyzed.

Bone marrow-derived dendritic cell generation. To generate mouse bone marrow-derived dendritic cells (BMDCs), bone marrow was flushed from femurs and tibias of BALB/c mice.
Cells $\left(1 \times 10^{6}\right.$ cells $\left./ \mathrm{ml}\right)$ were plated for 5 days in RPMI-1640 medium (Sigma) containing 10\% fetal calf serum (Sigma), antibiotics $(100 \mathrm{U} / \mathrm{ml}$ of penicillin, $100 \mathrm{mg} / \mathrm{ml}$ of streptomycin), $10 \mathrm{mM}$ HEPES buffer and $5 \times 10^{-5} \mathrm{M} 2-\beta$ mercaptoethanol (CM, complete medium), and $5 \%$ culture supernatant from a cell line transfected with the murine GM-CSF gene (26) was used as a source of GM-CSF equal to $100 \mathrm{U} / \mathrm{ml} \mathrm{rmGM-}$ CSF. Every second day, the cultures were fed by half volume of the medium with addition of fresh CM with GM-CSF.

Pulsing of BMDCs with VLPs or Bcr-Abl peptides. Five days old BMDCs were seeded in 24-well plate at the concentration of $1 \times 10^{6}$ cells/well in RPMI medium without FCS. VLPs composed of VP1 only ( $5 \mu \mathrm{g} /$ well) or 18-mer or 9-mer Bcr-Abl peptide or irrelevant peptide $(10 \mu \mathrm{g} /$ well $)$ were added to the cultures and incubated at $37^{\circ} \mathrm{C}$ in a $5 \% \mathrm{CO}_{2}$ incubator. After $1 \mathrm{~h}, \mathrm{CM}$ was added to the cells and incubation continued for another 4-20 h. Control (unpulsed) DCs and pulsed dendritic cells (pDCs) were used as stimulatory cells. To control the BMDC activation state, the presence of surface markers CD86 and MHC II was analyzed by flow cytometry. BMDCs pulsed by lipopolysaccharide (LPS) and untreated BMDCs served as controls.

Cell proliferation analysis. Single-cell suspensions from the spleens of BALB/c mice were prepared in CM. Effector cells $\left(1.5 \times 10^{6}\right)$ were labeled with $2 \mu \mathrm{M}$ carboxyfluorescein diacetate succinimidyl ester (CFSE, Molecular Probes) and cultivated with pulsed or unpulsed DCs $\left(3 \times 10^{5}\right)$ in 48 -well plate for 4 days. Proliferation of $\mathrm{T}$ cells was evaluated by gradual CFSE dilution in dividing $\mathrm{T}$ cells by flow cytometry.

ELISPOT assay. Spleens from mice immunized with Bcr-Abl VLPs or VLPs composed of VP1 only (wt VLPs) or with PBS were homogenized 12 days after the last immunization and lymphocytes were separated as described (27). Pooled splenocytes were cultivated in vitro in the presence of $0.01 \mu \mathrm{g}$ of 9-mer Bcr-Abl peptide or 18-mer Bcr-Abl peptide. After 5 days, the response of IFN $\gamma$-producing cells was detected by ELISPOT as described previously (27). The spots were evaluated by the Eli.Scan ELISPOT Scanner (A.EL.VIS, Hannover, Germany).

Cytotoxicity assay using target cells labeled with two different CFSE intensities. Spleen cells from immunized mice were restimulated in vitro by 4-day incubation with DCs pulsed with VLPs or Bcr-Abl peptide and used as effector cells for in vitro cytotoxicity assay. VLP and Bcr-Abl peptide pulsed DCs were labeled with $0.6 \mu \mathrm{M}$ CFSE for 5 min (high intensity) and DCs pulsed with irrelevant peptide were labeled with $0.04 \mu \mathrm{M}$ CFSE for $3 \mathrm{~min}$ (low intensity). Both fractions were adjusted to a similar concentration, mixed at ratios 1:1 and used as target cells. Effector cells were mixed with target cells at ratios 100:1, 50:1, 25:1 and incubated in CM in 96-Uwell plate at $37^{\circ} \mathrm{C}$ in a $\mathrm{CO}_{2}$ incubator overnight. The decrease of high CFSE labeled target cells was determined by flow cytometry analysis.

Cytotoxicity assay using MHC class I Bcr-Abl specific pentamers. The assay was performed according to Handbook 
of Proimmune company. Single spleen cell suspensions from immunized and control mice were prepared. Splenocytes were depleted from red blood cells by 15 -min incubation with fresh ammonium chloride lysing solution $\left(0.15 \mathrm{M} \mathrm{NH}_{4} \mathrm{Cl}\right.$, $1 \mathrm{mM} \mathrm{KHCO}{ }_{3}, 0.1 \mathrm{mM}$ EDTA in distilled water) in the dark, at room temperature, washed with PBS and resuspended in wash buffer $\left(0.1 \%\right.$ BSA in PBS). Splenocytes $\left(2 \times 10^{6}\right)$ were washed with ice-cold wash buffer and resuspended in the residual volume $(\sim 50 \mu 1)$. Samples were incubated with $10 \mu \mathrm{l}$ of labeled pentamers for $10 \mathrm{~min}$ at room temperature shielded from light. Splenocytes were washed with $2 \mathrm{ml}$ of ice-cold wash buffer and resuspended in the residual volume. AntiCD8 and anti-CD19 antibodies were added and the samples were incubated on ice for 20 min in dark. Cells were washed twice with ice cold wash buffer, resuspended in residual volume and analyzed by flow cytometry.

Detection of specific VPl and Bcr-Abl antibodies. Maxisorp 96-well plates were coated overnight at $4^{\circ} \mathrm{C}$ with either $5 \mu \mathrm{g}$ VLPs or 18 -mer Bcr-Abl synthetic peptide in $100 \mu \mathrm{l}$ of PBS per well. Wells were washed with PBS/Tween-20 (0.1\%) and saturated with $200 \mu \mathrm{l}$ of $2 \%$ milk powder in PBS. After 2-h incubation at room temperature, the blocking solution was removed and wells were overlaid with $100 \mu \mathrm{l}$ of decreasing dilutions of sera from control or immunized mice and incubated for $1 \mathrm{~h}$ at room temperature. Anti-PyV-VP1 mouse monoclonal antibody or anti-Bcr-Abl rabbit polyclonal serum was used as positive control. Wells were washed 4-times with PBS/ Tween-20 $(0.1 \%)$ and secondary antibodies conjugated with horseradish peroxidase were added. After 1-h incubation at room temperature, wells were washed with PBS/Tween-20 $(0.1 \%)$ and overlaid with $100 \mu \mathrm{l}$ of substrate solution (ABTS, Sigma). The absorbance of each well was determined at $415 \mathrm{~nm}$.

\section{Results}

Preparation and characterisation of chimeric MPyV VLPS carrying epitope of Bcr-Abl fusion protein. Bcr-Abl VLPs carrying 171 amino acids covering the breakpoint region of the human Bcr-Abl protein (together with FLAG sequence) were prepared. FLAG-Bcr-Abl sequences were fused to the C-terminal part of MPyV minor capsid protein, VP3, which is responsible for its interaction with the central cavity of the pentamers formed by the major structural protein, VP1. The particles were produced in insect cells by expression of the fused FLAG-Bcr-Abl-tVP3 sequences and wild-type VP1 gene from a recombinant baculovirus. Schema of the particle structure is shown in Fig. 1A. The ability of the proteins to assemble into VLPs was determined by electron microscopy. The incorporation of the fused polypeptide into the capsids influenced their morphology as shown by negative staining of isolated particles (Fig. 1C). Chimeric VLPs were larger and more irregular in comparison with the wtVLPs composed of VP1 only, but their stability was not substantially affected. The presence of foreign epitopes fused with TVP3 in purified particles was proved by SDS-PAGE (Fig. 1Ba), Western blotting using anti-FLAG antibody, anti-VP2/3 antibody (Fig.1Bb, c) and immunoelectron microscopy using antiBcr-Abl polyclonal serum (Fig. 1D). As the epitopes were hidden inside the particles, they could be stained by immunoelectron microscopy only after their disassembly under the reducing conditions.

To estimate an average number of Bcr-Abl ${ }_{171}-\mathrm{tVP} 3$ molecules incorporated into each VLP, we measured densities of VP1 and Bcr-Abl ${ }_{171}-\mathrm{tVP} 3$ bands of purified VLPs separated on SDS-PAGE and stained with Coomassie brilliant blue. Theoretically, maximum 72 pentameres of VP1 are available for t-VP3 interactions in one virus particle. The number of Bcr-Abl ${ }_{171}-\mathrm{tVP} 3$ per particle estimated from densities and molecular weights of both proteins was 11.4.

The ability of isolated VLPs to adsorb on the cell surface and to be internalized by cells was proved by confocal microscopy of cells fixed and stained with anti-VP1 antibody after adsorption of VLPs on their surface at $0^{\circ} \mathrm{C}$ or after adsorption and $1-\mathrm{h}$ incubation at $37^{\circ} \mathrm{C}$. Morphological changes caused by incorporation of epitopes had no effect on the Bcr-Abl VLP assembly and stability and VLPs adsorbed to and entered cells efficiently as shown in Fig. 2.

Both intranasal and intraperitoneal immunization by chimeric VLPs induced high titres of anti-VPl antibodies but no anti-Bcr-Abl antibodies. Mice immunized as described in Materials and methods were bled on day 14 after the last immunization and the presence of specific anti-VP1 and anti-Bcr-Abl antibodies was determined by ELISA. In agreement with our previous results (20), high titers of specific IgG anti-VP1 antibodies were detected in all immunized mice (Fig. 3A). In addition, when mice were immunized intraperitoneally with four doses of VLPs, the titers of specific anti-VP1 antibodies were much higher than those of mice after twice intranasal administration (Fig. 3A, black triangles). However, no specific anti-Bcr-Abl IgG antibodies were developed in the sera of either intranasally or intraperitoneally immunized mice (Fig. 3B). Moreover, no specific anti-Bcr-Abl IgM antibodies were detected by ELISA as shown in Fig. 3C. Finally, synthetic Bcr-Abl peptide (18-mer) was administered intraperitoneally in CFA into mice. Neither specific antiBcr-Abl IgG nor IgM antibodies in the sera of immunized mice were detected (data not shown).

No activation of BMDCs by 18-mer and 9-mer Bcr-Abl peptides was detected. To investigate the ability of Bcr-Abl VLPs or 18-mer or 9-mer Bcr-Abl peptides to activate dendritic cells, the expression of CD86 and MHC II on pulsed DCs was analyzed. After $20 \mathrm{~h}$, cells were harvested and stained for CD86 and MHC class II and analyzed by flow cytometry. Increased expression of CD86 and MHC class II was observed when BMDCs were stimulated with Bcr-Abl VLPs. The level of expression of MHC II was higher than that induced by LPS (Fig. 4B). This result was expected and corresponds to our previous observation of high immunogenicity of MPyV wtVLPs (20). However, no increased expression of either CD86 or MHC II was observed on DCs pulsed with 18-mer or 9-mer Bcr-Abl peptides (Fig. 4).

VLP-pulsed DCs, but not Bcr-Abl peptide-pulsed DCs stimulated antigen specific proliferation of splenocytes derived from mice immunized by Bcr-Abl-VLPS. In the first experiments, mice were immunized intranasally by chimeric 


\section{Mouse polyomavirus Bcr-Abl-VLPs}

A

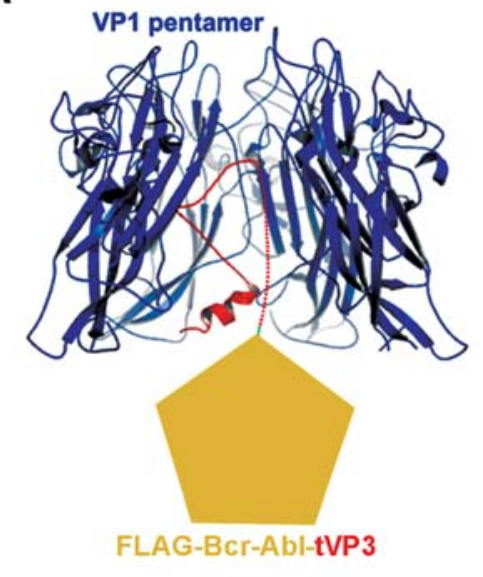

C

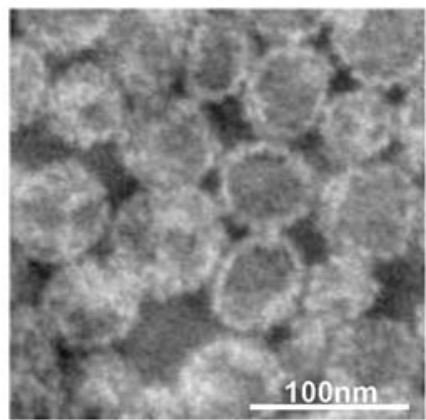

B

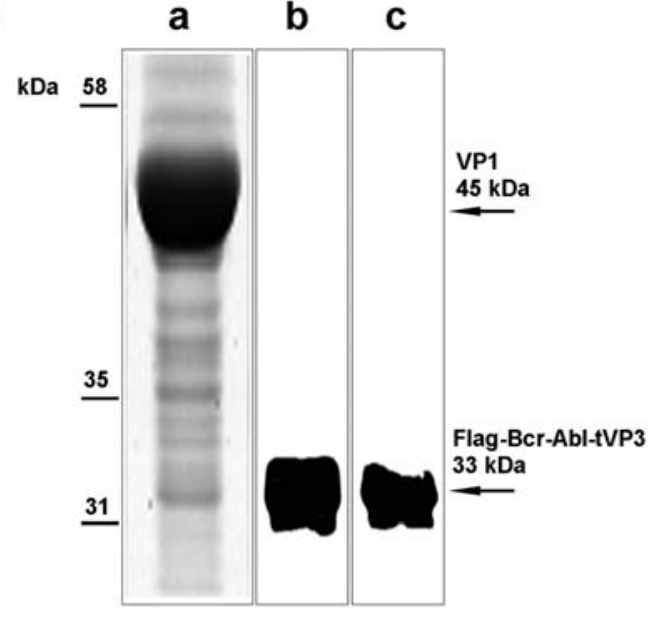

D

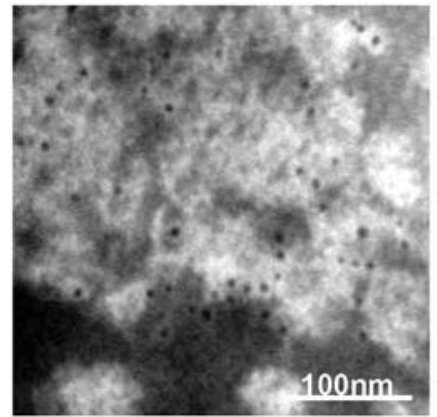

Figure 1. Structure and architecture of the analysed MPy VLPs carrying epitope of Bcr-Abl fusion protein. (A) View-through the pentamer composed of 5 molecules of VP1 protein (blue). C-terminal part of minor structural protein VP3 (red) interacts with central cavity of VP1 pentamer and is fused with Flag-Bcr-Abl epitope (illustrated as yellow pentagon). (Ba) Purified Bcr-Abl-VLPs separated on SDS-PAGE gel stained with Coomassie brilliant blue and (Bb and c) Western blotting of Bcr-Abl-VLPs using (b) mouse monoclonal anti-Flag antibody and (c) mouse monoclonal anti-VP2/3 antibody followed by goat anti-mouse IgG antibody conjugated with horse-radish peroxidase. (C) Electron microscopy and (D) immunoelectron microscopy with anti-Bcr-Abl rabbit polyclonal serum followed by goat anti-rabbit IgG antibody conjugated with $10 \mathrm{~nm}$ colloidal gold.
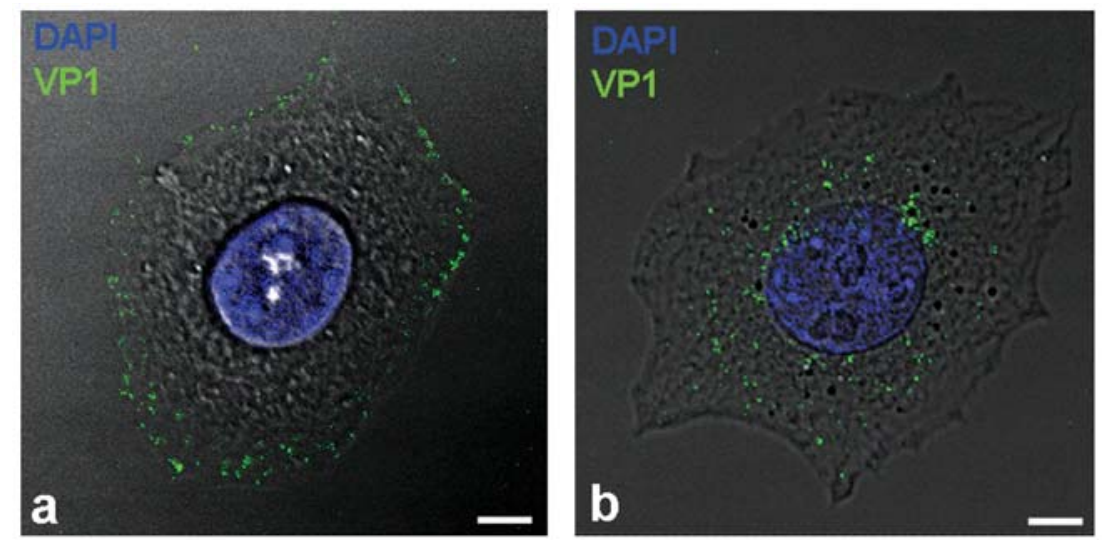

Figure 2. Adsorption (a) and following internalization (b) of Bcr-Abl-VLPs to the cells, sections from confocal microscope. Bcr-Abl-VLPs were adsorbed on the membrane of mouse fibroblasts $3 \mathrm{~T} 6$ and fixed $0 \mathrm{~min}$ or $1 \mathrm{~h}$ post-adsorption. The signal of VP1 protein (green) was visualised using anti-PyV VP1 mouse monoclonal antibody followed by Alexa Fluor-488 goat anti-mouse secondary antibody and examined by confocal microscope.

VLPs. Fourteen days after the second immunization, spleens from immunized and control mice were removed and single cell suspensions were used to study $\mathrm{T}$ cell-specific proliferative response. CFSE labeled spleen cells were stimulated for 4 days with three different types of BMDCs (unpulsed or pulsed with VLPs or pulsed with 9-mer Bcr-Abl peptide). The proliferative response (corresponded to CFSE diminution) was determined by flow cytometry. Fig. 5 shows VP1 specific proliferation of 


\section{A VP1-specific antibodies}

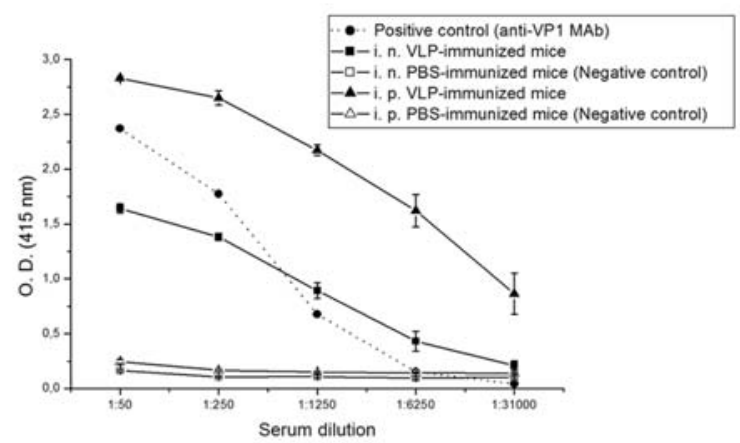

B

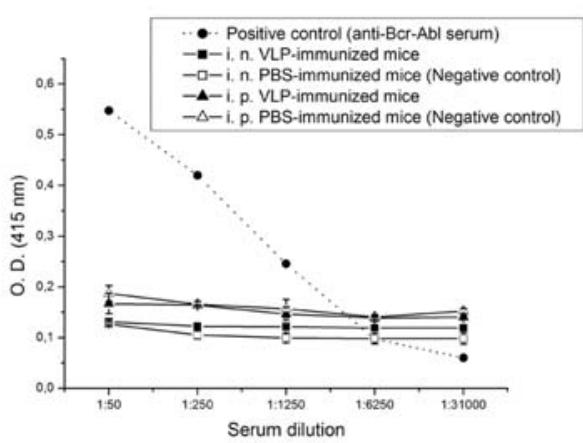

C Bcr-Abl-specific IgM antibodies

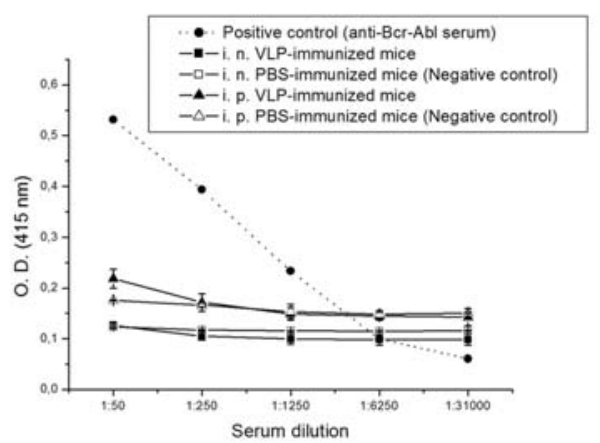

Figure 3. Specific anti-VP1 and anti-Bcr-Abl antibodies detected in the sera of control and immunized mice. (A) Anti-VP1 IgG antibodies in sera from intranasally (i.n.) and intraperitoneally (i.p.) PBS- (negative control) or Bcr-Abl-VLP-immunized mice. Anti-VP1 mouse monoclonal antibody served as positive control. (B) IgG and (C) IgM anti-Bcr-Abl antibodies in sera from intranasally (i.n.) and intraperitoneally (i.p.) PBS- (negative control) or Bcr-AblVLP-immunized mice. Anti-Bcr-Abl rabbit polyclonal serum was used as positive control.

\section{Activation of DCs by VLPs and Bcr-Abl peptides}

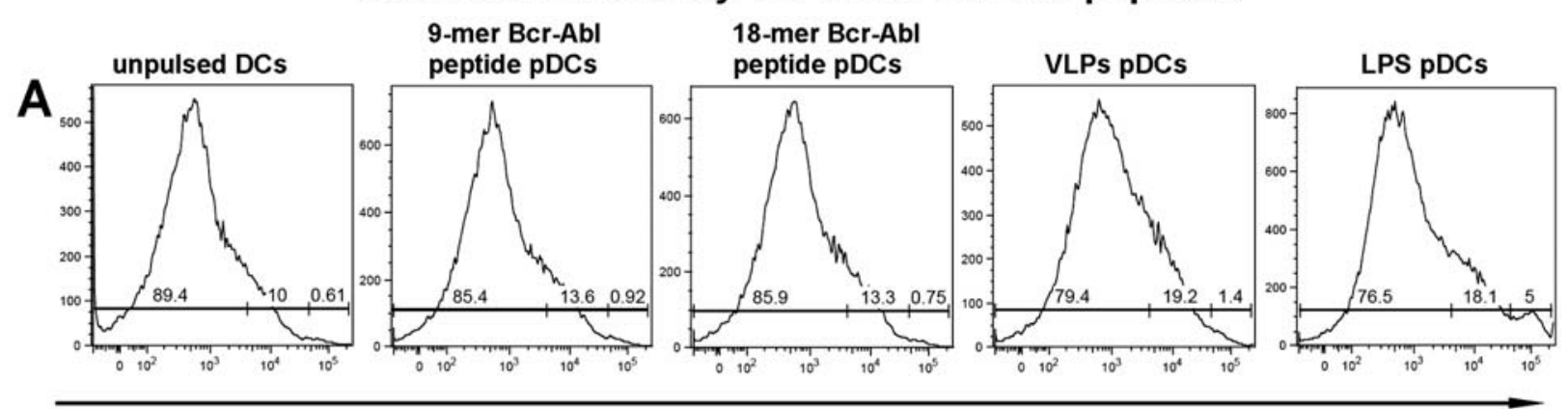

\section{Expression of CD86}

B

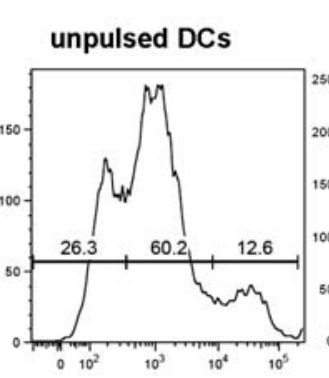

9-mer Bcr-Abl peptide pDCs

18-mer Bcr-Abl peptide pDCs
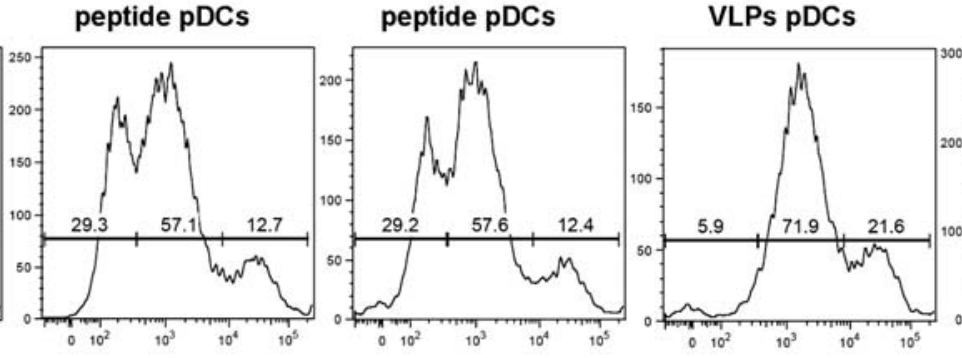

LPS pDCs

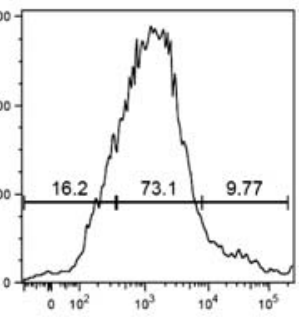

\section{Expression of MHC II}

Figure 4. Activation of dendritic cells by Bcr-Abl-VLPs and 18-mer and 9-mer Bcr-Abl peptides. Expression of CD86 (A) and MHC II (B) surface molecules by dendritic cells after pulsing by Bcr-Abl-VLPs, 18-mer or 9-mer Bcr-Abl peptides. Untreated DCs served as negative control and DCs pulsed with LPS was used as positive control. 


\section{Proliferative response of spleen cells}

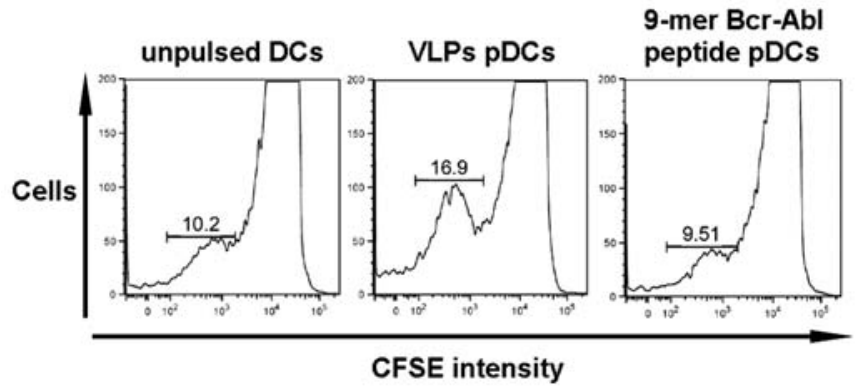

Figure 5. Proliferation of $\mathrm{T}$ cells from mice immunized intranasally by Bcr-Abl-VLPs. Spleen cell suspensions from immunized mice were restimulated with untreated DCs or DCs pulsed with Bcr-Abl-VLPs or Bcr-Abl peptide. Specific proliferative response corresponds to CFSE diminution. One representative experiment is shown.
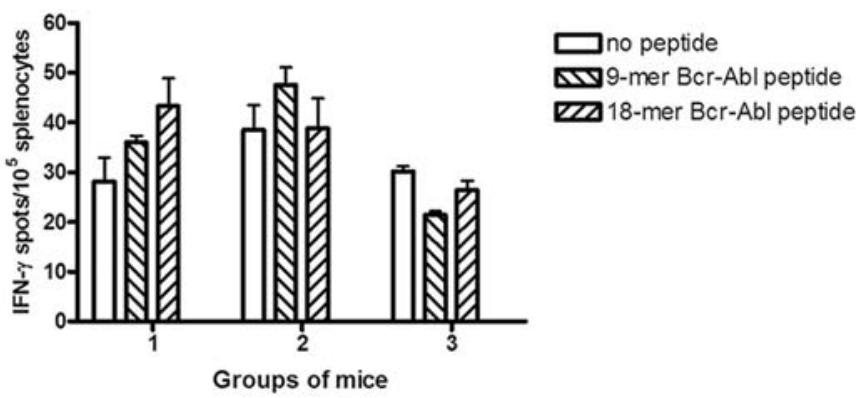

Figure 6. Secretion of IFN $\gamma$ by splenocytes restimulated with Bcr-Abl peptides. Three groups of mice (3 animals of each group) were immunized with Bcr-Abl-VLPs (1) or wtVLPs (2) or with PBS (3). IFN $\gamma$ production by splenocytes prepared 12 days after the last immunization was measured by ELISPOT.
T cells of mice immunized by Bcr-Abl-VLPs after stimulation of splenocytes with Bcr-Abl-VLP-pulsed DCs. However, no $\mathrm{Bcr}-\mathrm{Abl}$ specific proliferative response of $\mathrm{T}$ cells was observed when spleen cells from immunized mice were incubated with Bcr-Abl peptide pDCs (Fig. 5).

Bcr-Abl peptides do not induce INF $\gamma$ production in splenocytes derived from mice immunized by Bcr-Abl-VLPs. While MPyV-VLPs were shown to induce IFN $\gamma$ secretion in splenocytes of mice immunized by VLPs (after their restimulation by VLPs) (20), no significant increase of INF $\gamma$ production was detected in splenocytes of mice immunized by Bcr-Abl VLPs restimulated by 18 -mer or 9-mer Bcr-Abl peptides (Fig. 6).

VLPs carrying Bcr-Abl epitope do not induce specific BcrAbl cytotoxic activity of $T$ cells. Simultaneously with the proliferation experiment, the specific cytotoxic activity of $\mathrm{T}$ cells using target cells labeled with two different CFSE intensities (described in Materials and methods) was determined. Splenocytes were restimulated with Bcr-Abl peptide pulsed DCs for 4 days prior to cytotoxic assay. No Bcr-Abl specific cytotoxicity of $\mathrm{T}$ cells, measured by flow cytometry (as disappearance of DC pulsed with Bcr-Abl peptide) was observed (Fig. 7). For comparison VP1 specific cytotoxic activity was performed with two populations of target dendritic cells (pulsed with irrelevant peptide - low CSFE and pulsed with VP1-VLPs - high CSFE). The decrease of high CFSE labeled target cells was 5\% for ratio effectors: targets - 100:1.

In addition, another cytotoxic assay using MHC class I pentamers conjugated with Bcr-Abl restricted peptide was performed. Mice were immunized intranasally or intraperi-

\section{Cytotoxicity assay}

\section{Ratios of effector cells and target cells}

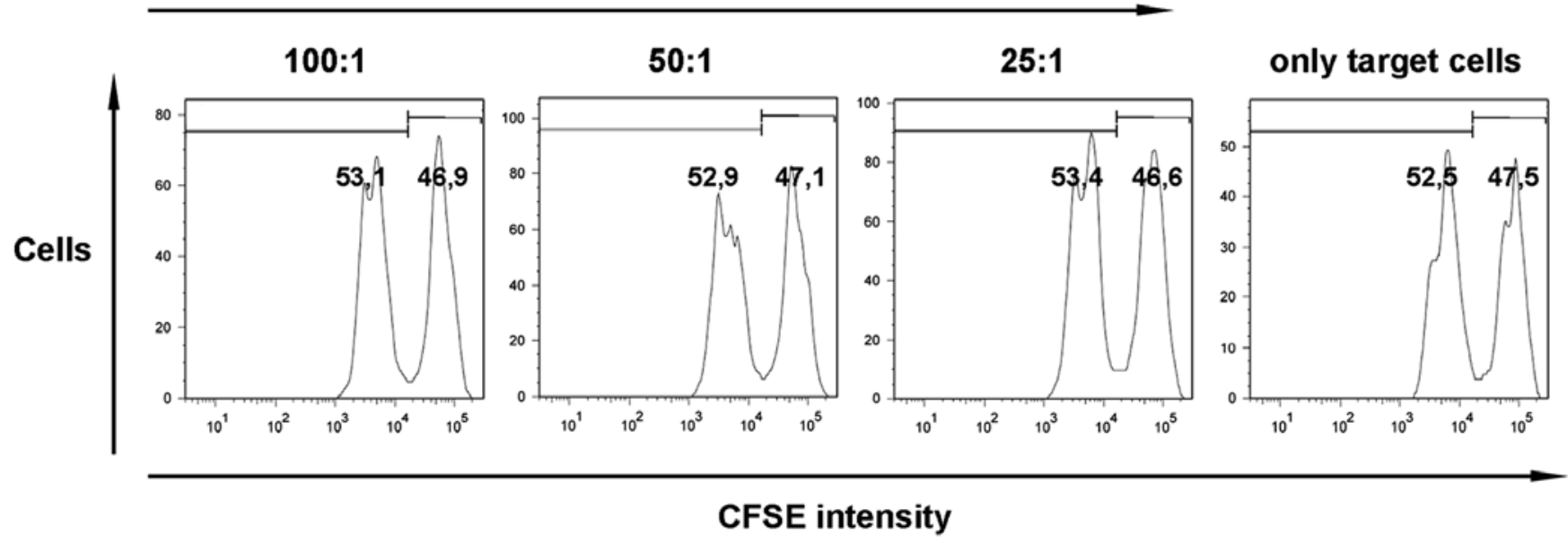

Figure 7. Cytotoxic response of spleen cells from control and intranasally immunized mice. Spleen cells from mice immunized by Bcr-Abl-VLPs were used as effector cells after their previous restimulation in vitro by dendritic cells pulsed with Bcr-Abl peptide. DCs pulsed with Bcr-Abl peptide (high CFSE intensity) and irrelevant peptide (low CFSE intensity) were mixed 1:1 and used as target cells with decreasing ratios of effector cells:target cells (100:1, 50:1, $25: 1)$. 


\section{Cytotoxicity assay using MHC class I Bcr-Abl specific pentamer staining}

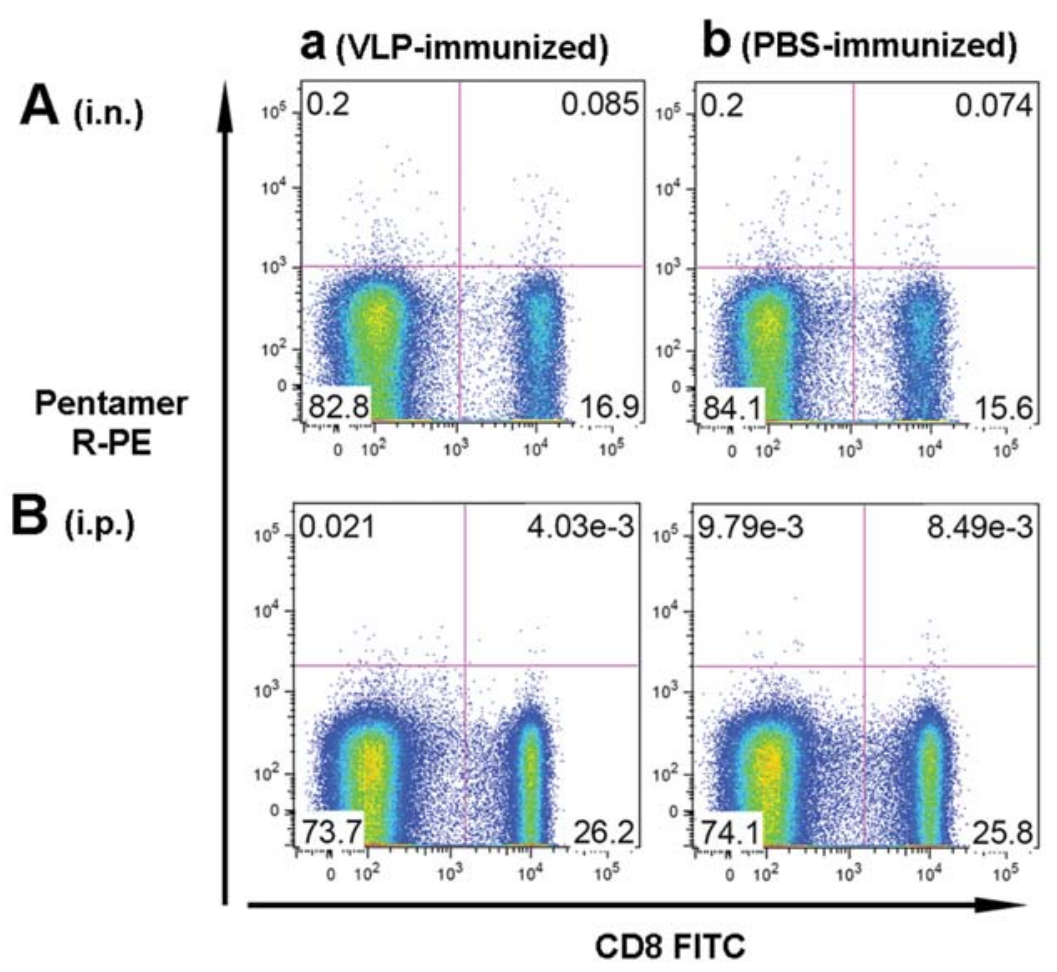

Figure 8. Detection of Bcr-Abl specific cytotoxic T lymphocytes in splenocytes of immunized mice. Mice were immunized intranasally (i.n., A) or intraperitoneally (i.p., B) with Bcr-Abl- VLPs (a). The presence of Bcr-Abl specific T lymphocytes (upper right quadrant of each graph) was determined using MHC class I Bcr-Abl specific pentamer staining. To exclude the background of unspecific binding of labeled pentamer, mice immunized intranasally (Ab) or intraperitoneally $(\mathrm{Bb})$ with $\mathrm{PBS}$ were involved in the experiment.

toneally as described in Materials and methods. Two weeks after the last immunization, spleens from immunized and control mice were removed and single-cell suspensions were prepared. Splenocytes were incubated with labeled MHC class I pentamers to determine specific Bcr-Abl cytotoxic T lymphocytes. Simultaneously, cells were stained for CD8 and CD19. Fig. 8 shows that mice immunized by Bcr-Abl VLPs did not develop any specific cytotoxic response after both intranasal (Fig. 8A) and intraperitoneal (Fig. 8B) antigen application.

\section{Discussion}

In this study, we have constructed chimeric VLPs derived from the mouse polyomavirus (MPyV) carrying an epitope of human fusion protein $\mathrm{p} 210^{\mathrm{Bcr}-\mathrm{Abl}}$ of chronic myeloid leukemia, Bcr-Abl-VLPs. Bcr-Abl fusion protein is intimately associated with malignant transformation as well as maintenance of the malignant phenotype in patients with CML and therefore it might be a potential $\mathrm{T}$ cell target. CD4 $\mathrm{T}$ cell response against epitopes derived directly from $\mathrm{Bcr}-\mathrm{Abl}$ joining region was reported in a mouse model. In that study, immunization of mice with 12 amino acid peptides spanning the junction region of b3a2 together with Freund's adjuvant elicited $\mathrm{T}$ cell responses (28). Some results of peptide vaccinations of CML patients have demonstrated that $\mathrm{b} 3 \mathrm{a} 2$ or $\mathrm{b} 2 \mathrm{a} 2$ breakpoint epitopes can activate CD4 T cells (29-31). Inspired by promising results of these groups, we were interested whether immune responses to the Bcr-Abl joining region can be stimulated when the epitope will be carried inside MPy VLPs. Feasibility of transfer of foreign peptides or proteins by MPy VLPs (anchored inside the particles with common C-terminus sequence of the minor capsid proteins) was proved in our previous studies with model EGFP protein $(13,20)$. Surprisingly, all our present effort to detect a cellular immune response after different types of administrations into mice failed. We have confirmed the incorporation of the Bcr-Abl derived polypeptide carrying the newly formed epitope inside the purified particles by several methods (Fig. 1B and D). Similar to EGFP, 171 amino acid long Bcr-Abl peptide inside the particles changed their morphology. VLPs lost their regular shape (Fig. 1), however, their stability was not substantially influenced and irregular chimeric VLPs were efficiently adsorbed to and internalized by host cells. In our previous report, MPy VLPs carrying entire EGFP instead of Bcr-Abl sequences were shown to be efficiently internalized and processed by dendritic cells (13).

Immunoelectron microscopy of disassembled VLPs proved the presence of Bcr-Abl breakpoint epitope inside VLPs. Theoretically, a possible number of epitope molecules carried by one VLP is 72 (one molecule for one VP1 pentamer). The ratio of intensities of VP1 and Bcr-Abl ${ }_{171}-\mathrm{tVP} 3$ bands on SDS PAGE suggests that average number of the epitope molecules in one particle is 11-12. Tegerstedt et al (17) found 
morphology of MPy VLPs carrying 683 amino acid long Her2/neu connected with the entire VP2, the longer one of two minor capsid proteins, not affected. However, the estimated number of Her2/neu molecules per 1 particle was very low (3 molecules per VLP). Surprisingly, these chimeric particles were able to efficiently protect $\mathrm{Balb} / \mathrm{c}$ mice from outgrowth of the Her2 expressing tumor (17).

As expected, both intranasally and intraperitoneally immunized mice developed high titers of anti-VP1 specific but not anti-Bcr-Abl specific antibodies. Similar findings were obtained with MPy VLPs carrying EGFP in our previous study (20) or the Her2/neu epitope (17). We detected very low presence of neutralizing antibodies against VP1 protein in the sera of intranasally immunized mice (20) suggesting the possibility of repeating administration of chimeric VLPs containing 'hidden' antigen without being neutralized.

We were not able to detect any anti-Bcr-Abl specific antibodies (neither IgG nor IgM) in the sera of mice immunized with Bcr-Abl breakpoint peptide (18-mer) in CFA. However, the same peptide conjugated with blue carrier immunogenic protein (Pierce, Rockford IL, USA) induced specific antibody response in rabbits (25). The rabbit anti-Bcr-Abl polyclonal serum was used for detection of epitope in immunoelectron microscopy and as positive control for ELISA. However, the titer of specific anti-Bcr-Abl antibodies was low (Fig. 3B and C) suggesting low immunogenicity of the applied peptide.

The most important component of immune response in development of anti-cancer vaccine is induction of specific cytotoxic T-cell immunity against a cancer antigen. To ascertain whether Bcr-Abl epitope delivered by MPy VLPs evokes any specific cytotoxic response, we applied two methods: in vitro cytotoxicity assay with CFSE-labeled target cells and highly sensitive cytotoxicity assay using MHC class I Bcr-Abl specific pentamers. Both techniques revealed that neither intranasal nor intraperitoneal administration induced cytotoxic activity of $\mathrm{T}$ cells. Moreover, in contrast to VLPpulsed DCs, Bcr-Abl peptide-pulsed DCs did not stimulate antigen specific proliferation of splenocytes derived from mice immunized by Bcr-Abl-VLPs. In contrast, Tegerstedt et $a l$, who immunized mice with MPy VLPs carrying Her2/neu antigen inside the particles, proved the presence of antiHer2/neu specific cytotoxic T-cells in mice after a single vaccination with VLPs (17). Moreover, the induced specific immunity was so high that mice were protected against a lethal dose of Her2-positive D2F2/E2 tumor cell challenge. They did not observe anti-Her2/neu positive cytotoxic activity after immunization of mice with Her2/neu protein (17). Also in our previous study (20), intranasal delivery of EGFP by MPy VLPs induced a stronger CD4+ ${ }^{+}$-cell response than administration of rEGFP alone.

Surprisingly, in therapeutically successful experiment of Tegerstedt et al (17), in vitro upregulation of DC maturation markers was not observed. However, we did observe upregulation of DC co-stimulatory molecules or other maturation markers (CD 86, MHCII) by Bcr-Abl VLPs in vitro. Also, in our previous experiments $(13,20)$, EGFP-VLPs induced production of IL-12 by DCs in vitro and spleen cells from EGFP-VLP immunized mice produced IL-2 and IFN $\gamma$ after treatment with DCs pulsed by EGFP-VLPs. Nevertheless, neither 18-mer nor restricted 9-mer Bcr-Abl breakpoint peptide activated DCs in vitro. They did not stimulate production of INF $\gamma$ in splenocytes from Bcr-Abl VLP-immunized mice. All these data suggest that Bcr-Abl breakpoint peptides, as well as longer portion of Bcr-Abl sequences carried by VLPs, are weak immunogens.

Recently, several groups have been working on the development of vaccine against chronic myeloid leukemia. In approximately $75 \%$ of cases the investigators failed to elicit in vitro sizable CTL responses to Bcr-Abl epitopes in healthy donors as well as in patients with CML and found that Bcr-Abl protein is apparently not an immunodominant antigen in CML $(32,33)$. Study of Abu-Eisha et al (34) showed that most normal subjects and CML patients developed no proliferative responses to the 23 -mer b3a2 fusion peptide. Moreover, no improvement was obtained by using mature autologous dendritic cells to present the peptide. Intracellular IFN $\gamma$ production by $\mathrm{CD}^{+}{ }^{+} \mathrm{T}$-cells was also not induced by the 23-mer b3a2 peptide (34).

On the other hand, with mouse model, the research group of Katsanis $(3,35,36)$ were able to induce Bcr-Abl specific cytotoxic activity in vivo by vaccination of mice with 12B1 tumor-derived chaperone-rich cell lysate (CRCL) (35) or by dendritic cells loaded with CRCL (36), or by liver CRCL (not leukemia derived) embedded with exogenous MHC class I restricted $\mathrm{Bcr}-\mathrm{Abl}$ peptide. In contrast to the CRCL vaccine, when MHC class I restricted Bcr-Abl peptide was administered to mice alone, survival was similar to those of negative control (PBS-immunized or liver CRCL Bcr-Abl peptide-free-immunized) (3). In our hands, no protection of mice immunized with Bcr-Abl-VLPs was detected when mice were challenged, each with $3 \times 10^{3} 12 \mathrm{~B} 1$ murine leukemia cells (37), 12 days after immunization (not shown).

In conclusion, although MPy VLPs proved to be strong inducers of immune responses in several experiments $(17,20)$ their unique adjuvant ability failed to support immune response against carried unique Bcr-Abl epitope. Recently, hypothesis appeared that not Bcr-Abl itself but genes that are up-regulated by the Bcr-Abl kinase activity may represent the crucial antigens for the induction of a cytotoxic T-cell response against CML cells $(33,38)$. Scheich et al $(38)$ demonstrated that the constitutively active kinase domain of Bcr-Abl has a key role in enhancing the immunogenicity of Bcr-Abl cells as the HLA class I-restricted T-cell responses were dominated by Bcr-Abl-regulated antigens, and not by Bcr-Abl itself. This hypothesis and results indicate new approaches in development of therapeutic vaccines in CML therapy.

\section{Acknowledgements}

We are grateful to Martin Marek for plasmid pFastBacDualVP1/tVP3 construction and to Michal Smahel for providing the pGD210 plasmid. We also thank Vincent Lucansky for selection of highly immunogenic epitopes within the amino acid sequence of Bcr-Abl fusion protein and Vojtech Zila and Jitka Stokrova for electron microscopy. This work was supported by projects MSM0021620858, LC545 and $1 \mathrm{M} 0508$ from the Ministry of Education, Youth, and Sport of the Czech Republic and by MZ0 UHKT 2005 and by IGA, NC 6957-3 of Grant Agency of the Ministry of Health of the Czech Republic. 


\section{References}

1. Pendergast AM, Muller AJ, Havlik MH, Maru Y and Witte ON: $\mathrm{BCR}$ sequences essential for transformation by the BCR-ABL oncogene bind to the ABL SH2 regulatory domain in a nonphosphotyrosine-dependent manner. Cell 66: 161-171, 1991.

2. Rojas JM, Knight K, Wang L and Clark RE: Clinical evaluation of BCR-ABL peptide immunisation in chronic myeloid leukaemia: results of the EPIC study. Leukemia 21: 2287-2295, 2007.

3. Kislin KL, Marron MT, Li G, Graner MW and Katsanis E: Chaperone-rich cell lysate embedded with BCR-ABL peptide demonstrates enhanced anti-tumor activity against a murine BCR-ABL positive leukemia. FASEB J 21: 2173-2184, 2008.

4. Bertolotti-Ciarlet A, Ciarlet M, Crawford SE, Conner ME and Estes MK: Immunogenicity and protective efficacy if rotavirus 2/6-virus-like particles produced by a dual baculovirus expression vector and administered intramusculary, intranasaly, or orally to mice. Vaccine 21: 3885-3900, 2003.

5. Casal JI: Use of parvovirus-like particles for vaccination and induction of multiple immune responses. Biotechnol Appl Biochem 29: 141-150, 1999.

6. Pan Q, He K and Huang K: Development of recombinant porcine parvovirus-like particles as an antigen carrier formed by the hybrid VP2 protein carrying immunoreactive epitope of porcine circovirus type 2. Vaccine 26: 2119-2126, 2008.

7. Forstová J, Krauzewicz N, Wallace S, Street AJ, Dilworth SM, Beard S and Griffin BE: Cooperation of structural proteins during late events in the life cycle of polyomavirus. J Virol 67 : 1405-1413, 1993.

8. Forstová J, Krauzewicz N, Sandig V, Elliott J, Palková Z, Strauss $M$ and Griffin BE: Polyoma virus pseudocapsids as efficient carriers of heterologous DNA into mammalian cells. Hum Gene Ther 6: 297-306, 1995.

9. Soeda E, Krauzewicz N, Cox C, Stokrová J, Forstová J and Griffin BE: Enhancement by polylysine of transient, but not stable, expression of genes carried into cells by polyoma VP1 pseudocapsids. Gene Ther 5: 1410-1419, 1998.

10. Montross L, Watkins S, Moreland RB, Mamon H, Caspar DL and Garcea RL: Nuclear assembly of polyomavirus capsids in insect cells expressing the major capsid protein VP1. J Virol 65: 4991-4998, 1991.

11. Salunke DM, Caspar DL and Garcea RL: Self-assembly of purified polyomavirus capsid protein VP1. Cell 46: 895-904, 1986.

12. Tsai B, Gilbert JM, Stehle T, Lencer W, Benjamin TL and Rapoport TA: Gangliosides are receptors for murine polyoma virus and SV40. EMBO J 22: 4346-4355, 2003.

13. Boura E, Liebl D, Spísek R, Fric J, Marek M, Stokrová J, Holán V and Forstová J: Polyomavirus EGFP-pseudocapsids: analysis of model particles for introduction of proteins and peptides into mammalian cells. FEBS Lett 579: 6549-6558, 2004.

14. Krauzewicz N, Cox C, Soeda E, Clark B, Rayner S and Griffin BE: Sustained ex vivo and in vivo transfer of a reporter gene using polyoma virus pseudocapsids. Gene Ther 7: 1094-1102, 2000.

15. Heidari S, Vlastos A, Ramqvist T, Clark B, Griffin BE, Garcia MI, Perez M, Amati P and Dalianis T: Immunization of T-cell deficient mice against polyomavirus infection using viral pseudocapsids or temperature sensitive mutants. Vaccine 20: 1571-1578, 2002.

16. Caparrós-Wanderley W, Clark B and Griffin BE: Effect of dose and long-term storage on the immunogenicity of murine polyomavirus VP1 virus-like particles. Vaccine 22: 352-361, 2004.

17. Tegerstedt K, Lindencrona JA, Curcio C, Andreasson K, Tullus C, Forni G, Dalianis T, Kiessling R and Ramqvist T: A single vaccination with polyomavirus VP1/VP2Her2 virus-like particles prevents outgrowth of HER-2/neu-expressing tumors. Cancer Res 65: 5953-5957, 2005.

18. Neugebauer M, Walders B, Brinkman M, Ruehland C, Schumacher T, Bertling WM, Geuther E, Reiser CO, Reichel C, Strich S and Hess J: Development of a vaccine marker technology: display of B cell epitopes on the surface of recombinant polyomavirus-like pentamers and capsoids induces peptidespecific antibodies in piglets after vaccination. Biotechnol J 1: 1435-1446, 2006.
19. Skrastina D, Bulavaite A, Sominskaya I, Kovalevska L, Ose V, Priede D, Pumpens P and Sasnauskas K: High immunogenicity of a hydrophilic component of the hepatitis B virus preS1 sequence exposed on the surface of three virus-like particle carriers. Vaccine 26: 1972-1981, 2008.

20. Fric J, Marek M, Hrusková V, Holán V and Forstová J: Cellular and humoral immune responses to chimeric EGFP-pseudocapsids derived from the mouse polyomavirus after their intranasal administration. Vaccine 26: 3242-3251, 2008.

21. Daley GQ, van Etten RA and Baltimore D: Induction of chronic myelogenous leukemia in mice by the P210bcr/abl gene of the Philadelphia chromosome. Science 247: 824-830, 1990.

22. Invitrogen: Bac/to/Bac Baculovirus Expression System, 2004.

23. O'Reilly DR, Miller LK and Luckow VA: Baculovirus Expression Vectors. A Laboratory Manual, W.H. Freeman and Co., New York, NY, 1992.

24. Hink WF: Established insect cell line from the cabbage looper, trichuplusia ni. Nature 226: 466-467, 1970.

25. Ludvíková V, Hamsíková E, Sobotková E, Lucansky V, Smahel M and Vonka V: Use of polyclonal rabbit antibodies for detection of the bcr-abl fusion zone in cells transfected with experimental bcr-abl DNA vaccines. Int J Oncol 27: 265-274, 2005.

26. Zal T, Volkmann A and Stockinger B: Mechanisms of tolerance induction in major histocompatibility complex class II-restricted $\mathrm{T}$ cells specific for a blood-borne self-antigen. J Exp Med 180: 2089-2099, 1994

27. Mackova J, Kutinova L, Hainz P, Krystofova J, Sroller V, Otahal P, Gabriel P and Nemeckova S: Adjuvant effect of dendritic cells transduced with recombinant vaccinia virus expressing HPV16-E7 is inhibited by co-expression of IL12. Int J Oncol 24: 1581-1588, 2004.

28. Chen W, Peace DJ, Rovira DK, You SG and Cheever MA: T-cell immunity to the joining region of $\mathrm{p} 210 \mathrm{BCR}-\mathrm{ABL}$ protein. Proc Natl Acad Sci USA 89: 1468-1472, 1992.

29. Pinilla-Ibarz J, Cathcart K, Korontsvit T, Soignet S, Bocchia M, Caggiano J, Lai L, Jimenez J, Kolitz J and Scheinberg DA: Vaccination of patients with chronic myelogenous leukemia with bcr-abl oncogene breakpoint fusion peptides generates specific immune responses. Blood 95: 1781-1787, 2000.

30. Cathcart K, Pinilla-Ibarz J, Korontsvit T, Schwartz J, Zakhaleva V, Papadopoulos EB and Scheinberg DA: A multivalent bcr-abl fusion peptide vaccination trial in patients with chronic myeloid leukemia. Blood 103: 1037-1042, 2004.

31. Maslak PG, Dao T, Gomez M, Chanel S, Packin J, Korontsvit T, Zakhaleva V, Pinilla-Ibarz J, Berman E and Scheinberg DA: A pilot vaccination trial of synthetic analog peptides derived from the BCR-ABL breakpoints in CML patients with minimal disease. Leukemia 22: 1613-1616, 2008.

32. Yotnda P, Firat H, Garcia-Pons F, Garcia Z, Gourru G, Vernant JP, Lemonnier FA, Leblond V and Langlade-Demoyen PJ: Cytotoxic $T$ cell response against the chimeric p210 BCR-ABL protein in patients with chronic myelogenous leukemia. Clin Invest 101: 2290-2296, 1998.

33. Grünebach F, Mirakaj V, Mirakaj V, Müller MR, Brümmendorf T and Brossart P: BCR-ABL is not an immunodominant antigen in chronic myelogenous leukemia. Cancer Res 66: 5892-5900, 2006.

34. Abu-Eisha HM, Butt NM, Clark RE and Christmas SE: Evidence that a BCR-ABL fusion peptide does not induce lymphocyte proliferation or cytokine production in vitro. Leuk Res 31: 1675-1681, 2007.

35. Zeng Y, Feng H, Graner MW and Katsanis E: Tumor-derived, chaperone-rich cell lysate activates dendritic cells and elicits potent antitumor immunity. Blood 101: 4485-4491, 2003.

36. Zeng Y, Graner MW, Thompson S, Marron M and Katsanis E: Induction of BCR-ABL-specific immunity following vaccination with chaperone-rich cell lysates derived from $\mathrm{BCR}-\mathrm{ABL}^{+}$tumor cells. Blood 105: 2016-2022, 2004.

37. McLauglin J, Chianese $\mathrm{E}$ and Witte $\mathrm{ON}$ : In vitro transfornation of immature hematopoietic cells by the P210 BCR/ABL oncogene product of the Philadelphia chromosome. Proc Natl Acad Sci USA 84: 6558-6562, 1987.

38. Scheich F, Duyster J, Peschel C and Bernhard H: The immunogenicity of Bcr-Abl expressing dendritic cells is dependent on the Bcr-Abl kinase activity and dominated by Bcr-Abl regulated antigens. Blood 110: 2556-2560, 2007. 